\title{
Supersymmetry in the Quark-Diquark and the Baryon-Meson Systems
}

\author{
S. Catto, Y. Choun
}

\begin{abstract}
A superalgebra extracted from the Jordan algebra of the 27 and $\overline{27} \mathrm{dim}$. representations of the group $E_{6}$ is shown to be relevant to the description of the quark-antidiquark system. A bilocal baryon-meson field is constructed from two quark-antiquark fields. In the local approximation the hadron field is shown to exhibit supersymmetry which is then extended to hadronic mother trajectories and inclusion of multiquark states. Solving the spin-free Hamiltonian with light quark masses we develop a new kind of special function theory generalizing all existing mathematical theories of confluent hypergeometric type. The solution produces extra "hidden" quantum numbers relevant for description of supersymmetry and for generating new mass formulas.
\end{abstract}

Keywords: supersymmetry, relativistic quark model.

\section{A colored supersymmetry scheme based on $S U(3)^{c} \times S U(6 / 1)$}

Algebraic realization of supersymmetry and its experimental consequences based on supergroups of type $S U(6 / 21)$ and color algebras based on split octonionic units $u_{i}(i=0, \ldots, 3)$ was considered in our earlier papers $[1,2,3,4,5]$. Here, we add to them the local color group $S U(3)^{c}$. We could go to a smaller supergroup having $S U(6)$ as a subgroup. With the addition of color, such a supergroup is $S U(3)^{c} \times S U(6 / 1)$. The fundamental representation of $S U(6 / 1)$ is 7 -dimensional which decomposes into a sextet and a singlet under the spin-flavor group. There is also a 28-dimensional representation of $S U(7)$. Under the $S U(6)$ subgroup it has the decomposition

$$
28=21+6+1
$$

Hence, this supermultiplet can accommodate the bosonic antidiquark and fermionic quark in it, provided we are willing to add another scalar. Together with the color symmetry, we are led to consider the $(3,28)$ representation of $S U(3) \times S U(6 / 1)$ which consists of an antidiquark, a quark and a color triplet scalar that we shall call a scalar quark. This boson is in some way analogous to the $s$ quarks. The whole multiplet can be represented by an octonionic $7 \times 7$ matrix $Z$ at point $x$.

$$
Z=\mathbf{u} \cdot\left(\begin{array}{cc}
\overline{\mathbf{D}}^{*} & \mathbf{q} \\
i \mathbf{q}^{T} \sigma_{2} & \mathbf{S}
\end{array}\right)
$$

Here $\mathbf{D}^{*}$ is a $6 \times 6$ symmetric matrix representing the antidiquark, $\mathbf{q}$ is a $6 \times 1$ column matrix, $\mathbf{q}^{T}$ is its transpose and $\sigma_{2}$ is the Pauli matrix that acts on the spin indices of the quark so that, if $q$ transforms with the $2 \times 2$ Lorentz matrix $L, \mathbf{q}^{T} i \sigma_{2}$ transforms with $L^{-1}$ acting from the right. Similarly we have

$$
Z^{c}=\mathbf{u}^{*} \cdot\left(\begin{array}{cc}
\mathbf{D} & i \sigma_{2} \mathbf{q}^{*} \\
\mathbf{q}^{\dagger} & \mathbf{S}^{*}
\end{array}\right)
$$

to represent the supermultiplet with a diquark and antidiquark. The mesons, exotic mesons and baryons are all in the bilocal field $Z(1) \otimes Z^{c}(2)$ which we expend with respect to the center of mass coordinates in order to represent color singlet hadrons by local fields. The color singlets $56^{+}$and $70^{-}$will then arise as shown in our earlier papers $[1,2,3]$.

Now the $(\bar{D} q)$ system belonged to the fundamental representation of the $S U(6 / 21)$ supergroup. But $Z$ belongs to the (28) representation of $S U(6 / 1)$ which is not its fundamental representation. Are there any fields that belong to the 7-dimensional representation of $S U(6 / 1)$ ? It is possible to introduce such fictitious fields as a 6 -dimensional spinor $\xi$ and a scalar $a$ without necessarily assuming their existence as particles. We put

$$
\xi=\mathbf{u}^{*} \cdot \boldsymbol{\xi}, \quad a=\mathbf{u}^{*} \cdot \mathbf{a},
$$


so that both $\xi$ and $a$ are color antitriplets. Let

$$
\lambda=\left(\begin{array}{c}
\xi \\
a
\end{array}\right), \quad \lambda^{c}=\left(\begin{array}{c}
\hat{\xi} \\
a^{*}
\end{array}\right)
$$

where

Consider the $7 \times 7$ matrix

$$
\hat{\xi}=\mathbf{u} \cdot\left(i \sigma_{2} \boldsymbol{\xi}^{*}\right), \quad a^{*}=\mathbf{u} \cdot \mathbf{a}^{*}
$$

$$
W=\lambda \lambda^{c \dagger}=\left(\begin{array}{cc}
\xi \hat{\xi}^{\dagger} & \xi a \\
a \hat{\xi}^{\dagger} & 0
\end{array}\right) .
$$

$W$ belongs to the 28-dimensional representation of $S U(6 / 1)$ and transforms like $Z$, provided the components of $\xi$ are Grassmann numbers and the components of $a$ are even (bosonic) coordinates. The identification of $Z$ and $W$ would give

$$
\mathbf{s}=\mathbf{a} \times \mathbf{a}=0, \quad \mathbf{q}_{\alpha}=\boldsymbol{\xi}_{\alpha} \times \mathbf{a}, \quad \boldsymbol{D}_{\alpha \beta}^{*}=\boldsymbol{\xi}_{\alpha} \times \boldsymbol{\xi}_{\beta}
$$

A scalar part in $W$ can be generated by multiplying two different (7) representations. The $56^{+}$baryons form the color singlet part of the 84-dimensional representation of $S U(6 / 1)$ while its colored part consists of quarks and diquarks.

Now consider the octonionic valued quark field $q_{A}^{i}$, where $i=1,2,3$ is the color index and $A$ stands for the pair $(\alpha, \mu)$ with $\alpha=1,2$ being the spin index and $\mu=1,2,3$ the flavor index. (If we have $N$ flavors, $A=1, \ldots, N)$. As before

$$
q_{A}=u_{i} q_{A}^{i}=\mathbf{u} \cdot \mathbf{q}_{A}
$$

Similarly the diquark $D_{A B}$ which transforms like a color antitriplet is

$$
D_{A B}=q_{A} q_{B}=q_{B} q_{A}=\epsilon_{i j k} u_{k}^{*} q_{A}^{i} q_{B}^{j}=\mathbf{u}^{*} \cdot \mathbf{D}_{A B}
$$

We note, once again, that because $q_{A}^{i}$ are anticommuting fermion operators, $D_{A B}$ is symmetric in its two indices. The antiquark and antidiquark are represented by

and

$$
\begin{gathered}
\bar{q}_{A}=\mathbf{u}^{*} \cdot \overline{\mathbf{q}}_{A}, \\
\bar{D}_{A B}=\mathbf{u} \cdot \overline{\mathbf{D}}_{A B},
\end{gathered}
$$

respectively. If we have 3 flavors $q_{A}$ has 6 components for each color while $D_{A B}$ has 21 components.

At this point let us study the system $\left(q_{A}, \overline{\mathbf{D}}_{B C}\right)$ consisting of a quark and an antidiquark, both color triplets. There are two possibilities: we can regard the system as a multiplet belonging to the fundamental representation of a supergroup $S U(6 / 21)$ for each color, or as a higher representation of a smaller supergroup. The latter possibility is more economical. To see what kind of supergroup we can have, we imagine that both quarks and diquarks are components of more elementary quantities: a triplet fermion $f_{A}^{i}$ and a boson $C^{i}$ which is a triplet with respect to the color group and a singlet with respect to $S U(2 N)(S U(6))$ for three flavors). The $f_{A}^{i}$ is taken to have baryon number $1 / 3$ while $C$ has baryon number $-2 / 3$. The system

$$
q_{A}^{i}=\epsilon_{i j k} \bar{f}_{A}^{j} \bar{C}^{k}
$$

will be a color triplet with baryon number $1 / 3$. It can therefore represent a quark. We can write

$$
q_{A}=\mathbf{u} \cdot \mathbf{q}_{A}=\left(\mathbf{u}^{*} \cdot \overline{\mathbf{f}}_{A}\right)\left(\mathbf{u}^{*} \cdot \overline{\mathbf{C}}\right)
$$

With two anti-f fields we can form bosons that have the same quantum numbers as antidiquarks:

$$
\bar{D}_{A B}=\mathbf{u} \cdot \overline{\mathbf{D}}_{A B}=\left(\mathbf{u}^{*} \cdot \overline{\mathbf{f}}_{A}\right)\left(\mathbf{u}^{*} \cdot \overline{\mathbf{f}}_{B}\right)
$$

In this case the basic multiplet is $\left(\mathbf{f}_{A}, \mathbf{C}\right)$ which belongs to the fundamental representation of $S U(6 / 1)$ for each color component. The complete algebra to consider is $S U(3)^{c} \times S U(6 / 1)$ and the basic multiplet corresponds to the representation $(3,7)$ of this algebra. Let

$$
F=\left(\begin{array}{c}
f_{1} \\
f_{2} \\
\vdots \\
f_{6} \\
C
\end{array}\right), \quad f_{A}=\mathbf{u} \cdot \mathbf{f}_{A}, \quad C=\mathbf{u} \cdot \mathbf{C}
$$


We write $f^{1}, f^{2}$, etc., as

$$
f^{1}=\left(\begin{array}{c}
f_{1}^{1} \\
f_{2}^{1} \\
\vdots \\
f_{6}^{1}
\end{array}\right), \quad f^{2}=\left(\begin{array}{c}
f_{1}^{2} \\
f_{2}^{2} \\
\vdots \\
f_{6}^{2}
\end{array}\right), \quad \ldots
$$

Combining two such representations and writing $\overline{\mathbf{X}}=\mathbf{F} \times \mathbf{F}^{T}$ we have

$$
\bar{X}=\mathbf{u}^{*} \cdot \overline{\mathbf{X}}=\left(\begin{array}{c}
f^{1} \\
C^{1}
\end{array}\right) \quad\left(\begin{array}{ll}
f^{2 T} & C^{2}
\end{array}\right)-\left(\begin{array}{c}
f^{2} \\
C^{2}
\end{array}\right)\left(\begin{array}{ll}
f^{1 T} & C^{1}
\end{array}\right)
$$

Further making the identifications

$$
\mathbf{u}^{*} \cdot \mathbf{D}_{11}=2 f_{1}^{1} f_{1}^{2}, \quad \mathbf{u}^{*} \cdot \mathbf{D}_{12}=f_{1}^{1} f_{2}^{2}-f_{1}^{2} f_{2}^{1}, \quad \text { etc. }
$$

and

$$
\mathbf{u}^{*} \cdot \overline{\mathbf{q}}_{1}=f_{1}^{1} C^{2}-f_{1}^{2} C^{1}, \quad \mathbf{u}^{*} \cdot \overline{\mathbf{q}}_{2}=f_{2}^{1} C^{2}-f_{2}^{2} C^{1}, \quad \text { etc. },
$$

we see that $\bar{X}$ has the structure

$$
\bar{X}=\mathbf{u}^{*} \cdot \overline{\mathbf{X}}=\mathbf{u}^{*} \cdot\left(\begin{array}{cccc}
\mathbf{D}_{11} & \ldots & \mathbf{D}_{16} & \overline{\mathbf{q}}_{1} \\
\mathbf{D}_{12} & \ldots & \mathbf{D}_{26} & \overline{\mathbf{q}}_{2} \\
\mathbf{D}_{13} & \ldots & \mathbf{D}_{36} & \overline{\mathbf{q}}_{3} \\
\mathbf{D}_{14} & \ldots & \mathbf{D}_{46} & \overline{\mathbf{q}}_{4} \\
\mathbf{D}_{15} & \ldots & \mathbf{D}_{56} & \overline{\mathbf{q}}_{5} \\
\mathbf{D}_{16} & \ldots & \mathbf{D}_{66} & \overline{\mathbf{q}}_{6} \\
-\overline{\mathbf{q}}_{1} & \ldots & -\overline{\mathbf{q}}_{6} & 0
\end{array}\right)
$$

The 27 dimensional representation decomposes into $21+\overline{6}$ with respect to its $S U(6)$ subgroup.

Consider now an antiquark-diquark system at point $x_{1}=x-\frac{1}{2} \xi$ and another quark-antidiquark system at point $x_{1}=x+\frac{1}{2} \xi$, and take the direct product of $X\left(x_{1}\right)$ and $X\left(x_{2}\right)$. We see that we get

$$
\left(D_{A B}\left(x_{1}\right), \bar{q}_{D}\left(x_{1}\right)\right) \otimes\left(\bar{D}_{E F}\left(x_{2}\right), q_{C}\left(x_{2}\right)\right)
$$

consisting of the pieces

$$
H\left(x_{1}, x_{2}\right)=\left(\begin{array}{cc}
\bar{q}_{D}\left(x_{1}\right) q_{C}\left(x_{2}\right) & D_{A B}\left(x_{1}\right) q_{C}\left(x_{2}\right) \\
\bar{q}_{D}\left(x_{1}\right) \bar{D}_{E F}\left(x_{2}\right) & D_{A B}\left(x_{1}\right) \bar{D}_{E F}\left(x_{2}\right)
\end{array}\right)
$$

The diagonal pieces are bilocal fields representing color singlet $1+35$ mesons and $1+35+405$ exotic mesons, respectively, with respect to the subgroup $S U(3)^{c} \times S U(6)$ of the algebra. The off diagonal pieces are color singlets that are completely symmetrical with respect to the indices $(A B C)$ and $(D E F)$. They correspond to baryons and antibaryons in the representations 56 and $\overline{56}$ respectively of $S U(6)$.

We can define the baryonic components $F_{A B C}$ as

$$
F_{A B C}=-\frac{1}{2}\left\{D_{A B}, q_{C}\right\}
$$

and evaluate its components:

$$
D_{A B} q_{C}=\left(u_{1}^{*}\left(q_{A}^{2} q_{B}^{3}+q_{B}^{2} q_{A}^{3}\right)+u_{2}^{*}\left(q_{A}^{3} q_{B}^{1}+q_{B}^{3} q_{A}^{1}\right)+u_{3}^{*}\left(q_{A}^{1} q_{B}^{2}+q_{B}^{1} q_{A}^{2}\right)\right) \times\left(u_{1} q_{C}^{1}+u_{2} q_{C}^{2}+u_{3} q_{C}^{3}\right)
$$

This becomes

$$
D_{A B} q_{C}=-u_{0}^{*}\left(\left(q_{A}^{2} q_{B}^{3}+q_{B}^{2} q_{A}^{3}\right) q_{C}^{1}+\left(q_{A}^{3} q_{B}^{1}+q_{B}^{3} q_{A}^{1}\right) q_{C}^{2}+\left(q_{A}^{1} q_{B}^{2}+q_{B}^{1} q_{A}^{2}\right) q_{C}^{3}\right)
$$


Similarly the $q_{C} D_{A B}$ part is

$$
q_{C} D_{A B}=-u_{0}\left(\left(q_{A}^{2} q_{B}^{3}+q_{B}^{2} q_{A}^{3}\right) q_{C}^{1}+\left(q_{A}^{3} q_{B}^{1}+q_{B}^{3} q_{A}^{1}\right) q_{C}^{2}+\left(q_{A}^{1} q_{B}^{2}+q_{B}^{1} q_{A}^{2}\right) q_{C}^{3}\right)
$$

Since $u_{0}+u_{0}^{*}=1$, we have

$$
F_{A B C}=-\frac{1}{2}\left\{D_{A B}, q_{C}\right\}=\left(q_{A}^{1} q_{B}^{2}+q_{B}^{1} q_{A}^{2}\right) q_{C}^{3}+\left(q_{A}^{2} q_{B}^{3}+q_{B}^{2} q_{A}^{3}\right) q_{C}^{1}+\left(q_{A}^{3} q_{B}^{1}+q_{B}^{3} q_{A}^{1}\right) q_{C}^{2}
$$

which is completely symmetric with respect to indices $(A B C)$, corresponding to baryons.

In the limit $x_{2}-x_{1}=\xi \rightarrow 0, H$ can be represented by a local supermultiplet with dimension $2 \times 56+$ $2(1+35)+405=589$ of the original algebra. This representation includes 56 baryons, antibaryons, mesons and $q^{2} \bar{q}^{2}$ exotic mesons. It is empirically well known for many years that all light-quark hadrons lie upon linear Regge trajectories. Relation between linear trajectories, linear confinement and relativistic dynamics had been well studied. Massless quarks bound by a linear confinement potential generate a family of parallel Regge trajectories. Regge slopes and daughter spacings depend on the Lorentz nature and other properties of the interaction. These are well known also through numerical studies (exact solutions) to the massless spinless Salpeter wave equation and the quantized straight string.

A generalized second order equation with a normalized wave function including quark mass $m$ is given by [6] for $H^{2}$ :

$$
H^{2}=4\left[\left(m+\frac{1}{2} b r\right)^{2}+P_{r}^{2}+\frac{l(l+1)}{r^{2}}\right] ; \quad P_{r}^{2}=-\frac{\partial^{2}}{\partial r^{2}}-\frac{2}{r} \frac{\partial}{\partial r}
$$

and Schrödinger equation is

$$
\frac{\partial^{2} \psi}{\partial r^{2}}+\frac{2}{r} \frac{\partial \psi}{\partial r}+\left(\frac{E^{2}}{4}-\frac{1}{4} b^{2}\left(r+\frac{2 m}{b}\right)^{2}-\frac{l(l+1)}{r^{2}}\right) \psi=0
$$

We have developed [7] a new solution for the normalized wave function (including the small mass $m$ ):

$$
\begin{aligned}
\psi_{n_{r}, n_{\zeta}, l, \bar{m}}(r, \theta, \phi)= & \left(\frac{\left(n_{r}-1\right) !(2 l+2) !\left(n_{r}+l-\frac{1}{2}\right) ! \sqrt{\pi}}{(2 b)^{l+\frac{2}{3}}(l+1) !\left(l+\frac{1}{2}\right) !}-\right. \\
& m\left\{\sum_{p=0}^{n_{r}-1} \frac{2^{l+2}(p+l+1) !}{b^{l+2} p !}\left(\frac{\left(n_{r}-1\right) !\left(\frac{1}{2}\right) !}{\left(n_{r}-p-1\right) !\left(p-n_{r}+\frac{3}{2}\right) !}\right)^{2}+\right. \\
& \sum_{n=0}^{n_{r}-1} \frac{\left(n_{r}-1\right) !\left(n_{r}+l-\frac{1}{2}\right) !\left(n-\frac{1}{2}\right) !(n+l) !(2 n+l+1)}{n !\left(-\frac{1}{2}\right) !\left(n_{r}-n-1\right) !\left(n+l+\frac{1}{2}\right) !} \times \\
& \left.\left.\sum_{k=n}^{n_{\zeta}-1} \frac{(-1)^{n_{r}+k} 2^{n+l+2}\left(-\frac{1}{2}\right) !\left(n_{\zeta}-n-1\right) !(n+k+l+1) !\left(n+k+\frac{1}{2}\right) !}{b^{n+l+2}\left(k+\frac{1}{2}\right) !\left(n_{\zeta}-k-1\right) !(k+l+1) !\left(k+n-n_{r} \frac{3}{2}\right) !}\right\}\right)^{-\frac{1}{2}} \times \\
& r^{l} e^{-\frac{b}{4}\left(r+\frac{2 m}{b}\right)^{2}} \sum_{n=0}^{n} \frac{(-1)^{n}\left(n_{r}-1\right) !\left(n_{r}+l-\frac{1}{2}\right) !}{n !\left(n_{r}-n-1\right) !\left(n+l+\frac{1}{2}\right.}\left(\frac{1}{2} b r^{2}\right)^{n} \times \\
& \left\{1+m r \sum_{k=0}^{n_{\zeta}-n-1} \frac{(-1)^{k}(n+l) !(2 n+l+1)\left(n_{\zeta}-n-1\right) !\left(n-\frac{1}{2}\right) !}{2\left(k+n+\frac{1}{2}\right) !\left(n_{\zeta}-k-n-1\right) !(k+n+l+1) !}\left(\frac{1}{2} b r^{2}\right)^{k}\right\} \times \\
& Y_{l}^{\bar{m}}(\theta, \phi)
\end{aligned}
$$

In the above equation

$$
\sum_{n=0}^{n} \frac{(-1)^{n}\left(n_{r}-1\right) !\left(n_{r}+l-\frac{1}{2}\right) !}{n !\left(n_{r}-n-1\right) !\left(n+l+\frac{1}{2}\right.}\left(\frac{1}{2} b r^{2}\right)^{n}=F_{n}^{|\alpha|}\left(|\alpha|=n_{r}-1, \gamma=l+\frac{3}{2} ; z=\frac{1}{2} b r^{2}\right)
$$

This last expression is the confluent hypergeometric function. For this reason we call Eq. (31) as Grand Confluent hypergeometric function. The $n_{r}$ is the radial quantum number. We give a name of $n_{\zeta}$ to an additional hidden radial quantum number that appears in our solution. In many of the special functions for 
second order differential equation there is only one eigenvalue, but when we include the small mass $m$, two eigenvalues are created. We already know that one eigenvalue is equal to

$$
E_{r}^{2}=4 b\left(l+2 n_{r}-\frac{1}{2}\right)
$$

This was shown in our earlier paper [6]. The second one, caused by small mass $m$, is equal to

$$
E_{a h}^{2}=4 b\left(l+2 n_{\zeta}+\frac{1}{2}\right)
$$

Typical wave functions has only three quantum numbers. But this wave function has four quantum numbers which are $n_{r}, n_{\zeta}, l$, and $\bar{m}$. When we let the small mass $m$ equal to zero, then Eq. (31) simply becomes

$$
\psi_{n_{r}, n_{\zeta}, l, \bar{m}}(r, \theta, \phi)=\left(\frac{\left(n_{r}-1\right) !(2 l+2) !\left(n_{r}+l-\frac{1}{2}\right) ! \sqrt{\pi}}{(2 b)^{l+\frac{2}{3}}(l+1) !\left(l+\frac{1}{2}\right) !}\right)^{-\frac{1}{2}} r^{l} e^{-\frac{b}{4} r^{2}} F_{n}^{|\alpha|} Y_{l}^{\bar{m}}(\theta, \phi)
$$

where $F_{n}^{|\alpha|}=F_{n}^{|\alpha|}\left(|\alpha|=n_{r}-1, \gamma=l+\frac{3}{2} ; z=\frac{1}{2} b r^{2}\right)$

Eq. (35) is exactly the same as our previous wave function when the small mass $m$ is neglected. Our generalized solution gives rise to new mass formulas in remarkable agreement with experiments which will be described in our forthcoming publications. We believe that the new special functions we created will have great many uses in physics and other areas of sciences.

\section{Acknowledgement}

This work was supported in part by DOE contracts No. DE-AC-0276 ER 03074 and 03075 . One of us (SC) thanks Dean Jeffrey Peck for the travel fellowship and Professor Cestmir Burdik for the kind invitation to this conference to present this paper.

\section{References}

[1] Catto, S., Gürsey, F.: Nuovo Cim. 86 A, 201 (1985).

[2] Catto, S., Gürsey, F.: Nuovo Cim. 99A, 685 (1988).

[3] Catto, S.: Miyazawa Supersymmetry. In H. Sakai, B. F. Gibson (Eds.): New Facets of Three Nucleon Force. (2008) AIP 1011.

[4] Catto, S.: Scalar mesons, Multiquark States and Supersymmetry. In G. Rupp, B. Hiller, F. Kleefield (Eds.) Workshop on Scalar Mesons and Related Topics. (2008) AIP 1030.

[5] Catto, S.: Effective Supersymmetry Based on $S U(3)^{c} \times S$ Superalgebra. In V. Dobrev (ED.) Lie Theory and Its Applications in Physics. 2010.

[6] Catto, S., Cheung, H. Y., Gürsey, F.: Mod. Phys. Lett. A 383485 (1991).

[7] Catto, S., Choun, Y.: In preparation.

Sultan Catto

E-mail: scatto@gc.cuny.edu

The Graduate School

The City University of New York and Baruch College

17 Lexington Avenue, New York, NY 10010

Physics Theory Group

The Rockefeller University

1230 York Avenue, New York, NY 10021-6399

Yoon Choun

The Graduate School

The City University of New York and Baruch College

17 Lexington Avenue, New York, NY 10010 